\title{
Discontinuous Galerkin Methods for Multi-Pantograph Delay Differential Equations
}

\author{
Kun Jiang $^{1}$, Qiumei Huang ${ }^{1, *}$ and Xiuxiu $\mathrm{Xu}^{2}$ \\ ${ }_{1}^{1}$ College of Applied Sciences, Beijing University of Technology, Beijing 100124, China \\ 2 School of Mathematical Sciences, Anhui University, Hefei 230031, China
}

Received 20 April 2019; Accepted (in revised version) 13 July 2019

\begin{abstract}
In this paper, the discontinuous Galerkin method is applied to solve the multi-pantograph delay differential equations. We analyze the optimal global convergence and local superconvergence for smooth solutions under uniform meshes. Due to the initial singularity of the forcing term $f$, solutions of multi-pantograph delay differential equations are singular. We obtain the relevant global convergence and local superconvergence for weakly singular solutions under graded meshes. The numerical examples are provided to illustrate our theoretical results.
\end{abstract}

AMS subject classifications: 65L60, 65L70

Key words: Multi-pantograph, discontinuous Galerkin method, global convergence, local superconvergence, weakly singular, graded meshes.

\section{Introduction}

This paper deals with the properties of the following linear multi-pantograph delay differential equation (MPDDE),

$$
\begin{aligned}
& u^{\prime}(t)=a(t) u(t)+\sum_{i=1}^{l} b_{i}(t) u\left(q_{i} t\right)+f(t), \quad t \in J:=[0, T] \\
& u(0)=u_{0}
\end{aligned}
$$

where $a(t), b_{i}(t)$ are continuous functions, $q_{i} \in(0,1),(i=1,2, \cdots, l)$ are delay coefficients.

As one of the most important mathematical models, MPDDE is widely used in many fields such as engineering, biology systems, physics and medicine. The study of the MPDDE has been a rapid development by many authors numerically and analytically

${ }^{*}$ Corresponding author.

Emails: jkmath@126.com (K. Jiang), qmhuang@bjut.edu.cn (Q. M. Huang), xuxiuxiu@emails.bjut.edu.cn (X.X. Xu) 
these years. Ishiwata [1] analyzed the attainable order of collocation method for neutral functional-differential equations with proportional delays. Li and Liu [2] used the Runge-Kutta method to solve the multi-pantograph delay differential equation. Taylor method was also used to solve multi-pantograph delay differential equations, such as the paper by Sezer et al. [3]. Brunner [4] applied the collocation method to the pantographtype Volterra functional equation with multiple delays and $\mathrm{Yu}$ [5] used the variational iteration method to solve the multi-pantograph delay differential equation, respectively. Feng [6] employed the homotopy perturbation method to solve multi-pantograph delay differential equations with variable coefficients. Lately, Geng and Qian [7] solved the singularly perturbed multi-pantograph delay differential equations based on the reproducing kernel space method. Komashynska et al. [8] used the residual power series method to solve a system of multi-pantograph delay differential equations. Davaeifar and Rashidinia [9] utilized collocation methods for a system of multi-pantograph type delay differential equations with variable coefficients and obtained the approximate solutions based on the Boubaker polynomials. Zheng et al. [10] developed a Legendrecollocation spectral method for the second order Volterra integro-differential equation with delay. Sedaghat et al. [11] provided a spectral method based on the operational matrices of the Legendre polynomials to solve neutral multi-pantograph delay differential equations.

The discontinuous Galerkin (DG) method was first proposed in [12] as a nonstandard finite element method for numerical solutions of neuron transport problems. Then DG methods are extensively used in solving partial differential equations and integral differential equations. DG methods are also successfully applied to delay differential equations and highlighted advantages compared with difference methods. Brunner et al. [13] used the DG method to solve delay differential equation with one proportional delay. Li et al. [21] applied DG method for delay differential equations with constant delay. Huang et al. [14] improved the global convergence by some accelerate techniques based on the local superconvergence results of DG solutions, and presented the $h p$-version of the DG method with nonlinear vanishing delays [15]. They also developed the continuous Galerkin (CG) methods for delay differential equations of pantograph type with uniform meshes [16] and quasi-geometric meshes [17,18].

In this paper, we intend to effectively employ the DG method to approximate smooth solutions of the multi-pantograph delay differential equations with uniform meshes. Due to the initial singularity of the forcing term $f$, solutions of multi-pantograph delay differential equations are singular. We also intend to get the relevant global convergence and local superconvergence of DG solutions for weakly singular multi-pantograph delay differential equations with graded meshes. For simplicity but without loss of generality, we consider the following special multi-pantograph delay differential equation case:

$$
\begin{aligned}
& u^{\prime}(t)=a(t) u(t)+b_{1}(t) u\left(q_{1} t\right)+b_{2}(t) u\left(q_{2} t\right)+f(t), \quad t \in J:=[0, T], \\
& u(0)=u_{0} .
\end{aligned}
$$

We analyze the optimal global convergence and local superconvergence of discontinuous 
Galerkin solutions for (1.2).

This paper is organized as follows. In Section 2, we describe the discontinuous Galerkin method for the multi-pantograph delay differential equation (1.2). In Sections 3 and 4 we present the convergence results of DG solutions for smooth and weakly singular cases respectively. Section 5 illustrates numerical examples to confirm our theoretical results. Conclusions are made in the last section.

\section{The discontinuous Galerkin method}

In this section, we introduce the DG method of the MPDDE (1.2). Let $J_{h}$ be a partition of the given interval $J=[0, T]$ that divide $J$ into $N$ subintervals $\left\{I_{n}\right\}_{n=1}^{N}$. Set

$$
0=t_{0}<t_{1}<\cdots<t_{N}=T,
$$

and

$$
I_{n}:=\left(t_{n-1}, t_{n}\right), \quad h_{n}:=t_{n}-t_{n-1} .
$$

We assume that the given functions $a, b_{1}, b_{2}$ in (1.2) are continuous on $J$. The corresponding discontinuous finite element space is defined as

$$
S_{m}^{(-1)}\left(J_{h}\right)=\left\{v \in L^{2}(J):\left.v\right|_{I_{n}} \in P_{m}, 1 \leq n \leq N\right\} .
$$

Comparing with CG, the main difference of DG method is that there is no continuous restrictions at the nodes $\left\{t_{n}\right\}_{n=0}^{N}$. Therefore, the left-hand and right-hand limits of the elements $v \in S_{m}^{(-1)}\left(J_{h}\right)$ play an important role in the DG method. They are defined by

$$
\begin{aligned}
& v_{n}^{+}:=\lim _{s \rightarrow 0, s>0} v\left(t_{n}+s\right), \quad 0 \leq n \leq N-1, \\
& v_{n}^{-}:=\lim _{s \rightarrow 0, s>0} v\left(t_{n}-s\right), \quad 1 \leq n \leq N,
\end{aligned}
$$

we denote $[v]_{n}:=v_{n}^{+}-v_{n}^{-}$as the jump across at the interior node $t_{n}$.

For the DG method, we are looking for an approximate solution $U \in S_{m}^{(-1)}\left(J_{h}\right)$ such that

$$
\begin{aligned}
& \sum_{n=1}^{N} \int_{I_{n}} U^{\prime}(t) v(t) d t+\sum_{n=1}^{N-1}[U]_{n} v_{n}^{+}+U_{0}^{+} v_{0}^{+} \\
= & u_{0} v_{0}^{+}+\sum_{n=1}^{N} \int_{I_{n}}\left[a(t) U(t)+b_{1}(t) U\left(q_{1} t\right)+b_{2}(t) U\left(q_{2} t\right)+f(t)\right] v(t) d t, \quad \forall v \in S_{m}^{(-1)}\left(J_{h}\right) .
\end{aligned}
$$

Suppose that $l_{n, 1}(t), \cdots, l_{n, m+1}(t)(s)$ are given basis functions on the subinterval $I_{n}$ and $L_{1}(s), \cdots, L_{m+1}(s)$ are the corresponding basis functions on $[0,1]$. The discontinuous Galerkin solution can be written as

$$
U_{n}(t)=\sum_{j=1}^{m+1} u_{n, j} l_{n, j}(t)=\sum_{j=1}^{m+1} u_{n, j} L_{j}\left(\frac{t-t_{n-1}}{h_{n}}\right) .
$$


It is obvious that the exact solution $u$ of MPDDE (1.2) also satisfies (2.1), that is

$$
\begin{aligned}
& \sum_{n=1}^{N} \int_{I_{n}} u^{\prime}(t) v(t) d t+\sum_{n=1}^{N-1}[u]_{n} v_{n}^{+}+u_{0}^{+} v_{0}^{+} \\
= & u_{0} v_{0}^{+}+\sum_{n=1}^{N} \int_{I_{n}}\left[a(t) u(t)+b_{1}(t) u\left(q_{1} t\right)+b_{2}(t) u\left(q_{2} t\right)+f(t)\right] v(t) d t, \quad \forall v \in S_{m}^{(-1)}\left(J_{h}\right) .
\end{aligned}
$$

Hence, subtracting (2.2) from (2.1), and setting $e:=u-U$, we obtain

$$
\begin{aligned}
B_{D G}(e, v):=\sum_{n=1}^{N} \int_{I_{n}}\left(e^{\prime}(t)-a(t) e(t)-b_{1}(t) e\left(q_{1} t\right)-b_{2}(t) e\left(q_{2} t\right)\right) v(t) d t \\
\quad+\sum_{n=1}^{N}[e]_{n-1} v_{n-1}^{+}=0 .
\end{aligned}
$$

From (2.3) we have that the DG error $e$ possesses the orthogonality property

$$
B_{D G}(e, v)=0, \quad \forall v \in S_{m}^{(-1)}\left(J_{h}\right) .
$$

The DG solution can be obtained on subinterval $I_{n},(n=1, \cdots, N)$ by solving the following system:

$$
\begin{aligned}
& \int_{I_{n}} U^{\prime}(t) v(t) d t+U_{n-1}^{+} v_{n-1}^{+} \\
= & U_{n-1}^{-} v_{n-1}^{+}+\int_{I_{n}}\left[a(t) U(t)+b_{1}(t) U\left(q_{1} t\right)+b_{2}(t) U\left(q_{2} t\right)+f(t)\right] v(t) d t, \quad \forall v \in \mathcal{P}_{m}\left(I_{n}\right) .
\end{aligned}
$$

Here, we let $U_{0}^{-}:=u_{0}$.

In order to write the detailed computational scheme of (2.4), we define some vectors

$$
\begin{aligned}
& \mathbf{g}_{1}:=\left(L_{1}(0), \cdots, L_{m+1}(0)\right)^{T}, \\
& \mathbf{f}_{n}:=\left(\int_{0}^{1} f\left(t_{n-1}+s h_{n}\right) L_{1}(s) d s, \cdots, \int_{0}^{1} f\left(t_{n-1}+s h_{n}\right) L_{m+1}(s) d s\right)^{T}, \\
& \mathbf{U}_{n}:=\left(u_{n, 1}, \cdots, u_{n, m+1}\right)^{T} \in \mathbb{R}^{m+1},
\end{aligned}
$$

and the following matrices (in $\mathbb{R}^{(m+1) \times(m+1)}$ )

$$
\begin{aligned}
& M:=\left(\int_{0}^{1} L_{j}^{\prime}(t) L_{i}(t) d t+L_{j}(0) L_{i}(0)\right)_{1 \leq i, j \leq m+1}, \\
& A_{n}:=\left(\int_{0}^{1} a\left(t_{n-1}+s h_{n}\right) L_{j}(s) L_{i}(s) d s\right)_{1 \leq i, j \leq m+1}, \\
& G:=\left(L_{j}(1) L_{i}(0)\right)_{1 \leq i, j \leq m+1} .
\end{aligned}
$$


The contributions of the delay terms $\int_{I_{n}} b_{i}(t) U\left(q_{i} t\right) v(t) d t,(i=1,2)$ are governed by certain relationships between the values $n$ and $q_{i}$ of the delay functions $q_{i} t$. Therefore, the analysis of these delay items are the most important steps in the whole computational form. We have the following three cases depending on the images $q_{i} t$ (assume $q_{1}>q_{2}$ ):

- For $n=1$, we call the complete overlap: for any $t \in I_{1}$ the images $q_{i} t,(i=1,2)$ lie in $I_{1}$.

- If $q_{1} t_{n}>t_{n-1},(n \geq 2)$, we call the partial overlap: for some $t \in I_{n}$ the images $q_{1} t$ are still in $I_{n}$, while for other (smaller) $t \in I_{n}$ we have $q_{1} t \notin I_{n}$.

- If $q_{1} t_{n} \leq t_{n-1},(n \geq 2)$, we call the non-overlap: the images $q_{i} t,\left(t \in I_{n}, i=1,2\right)$ no longer have any overlap with $I_{n}$.

We give a brief idea of how to get the computational form of the multi-pantograph equation (1.2) by using DG method.

(1) In the first subinterval $I_{1}$, the images $q_{1} t$ and $q_{2} t$ both lie in $I_{1}$. We define the matrix

$$
\begin{aligned}
& B_{1}^{I}:=\left(\int_{0}^{1} b_{1}(h s) L_{j}\left(q_{1} s\right) L_{i}(s) d s\right)_{1 \leq i, j \leq m+1^{\prime}} . \\
& B_{2}^{I}:=\left(\int_{0}^{1} b_{2}(h s) L_{j}\left(q_{2} s\right) L_{i}(s) d s\right)_{1 \leq i, j \leq m+1} .
\end{aligned}
$$

Then the vector $\mathbf{U}_{1}$ is determined by the solution of linear algebraic system

$$
\left(M-h_{1} A_{1}-h_{1} B_{1}^{I}-h_{1} B_{2}^{I}\right) \mathbf{U}_{1}=u_{0} \mathbf{g}_{1}+h_{1} \mathbf{f}_{1} .
$$

(2) If $q_{1} t_{n}>t_{n-1},(n \geq 2)$, in this phase, there is an integer $\theta_{1}$ such that $q_{1} t_{n-1} \in I_{\theta_{1}+1}$. Let $s_{0}^{*}=0$ and $0<s_{1}^{*}, \cdots, s_{n-1-\theta_{1}}^{*}<1$ satisfying $q_{1}\left(t_{n-1}+s_{k_{1}}^{*} h_{n}\right)=t_{\theta_{1}+k_{1}}$ for $k_{1}=1, \cdots, n-1-\theta_{1}$. Then we have

$$
s_{k_{1}}^{*}:=\left(\frac{t_{\theta_{1}+k_{1}}}{q_{1}}-t_{n-1}\right) / h_{n} \in(0,1), \quad k_{1}=1, \cdots, n-1-\theta_{1} .
$$

For $k_{1}=1, \cdots, n-1-\theta_{1}$, we define

$$
\begin{aligned}
& B_{n, k_{1}}^{I I}:=\left(\int_{s_{k_{1}-1}^{*}}^{s_{k_{1}}^{*}} b_{1}\left(t_{n-1}+s h_{n}\right) L_{j}\left(\frac{q_{1}\left(t_{n-1}+s h_{n}\right)-t_{\theta_{1}+k_{1}-1}}{h_{\theta_{1}+k_{1}}}\right) L_{i}(s) d s\right)_{1 \leq i, j \leq m+1}, \\
& B_{n, 2}^{I I}:=\left(\int_{s_{n-1-\theta_{1}}^{*}}^{1} b_{1}\left(t_{n-1}+s h_{n}\right) L_{j}\left(\frac{q_{1}\left(t_{n-1}+s h_{n}\right)-t_{n-1}}{h_{n}}\right) L_{i}(s) d s\right)_{1 \leq i, j \leq m+1} .
\end{aligned}
$$

(i) If $q_{2} t_{n}>t_{n-1}$, in a similar way, there is an integer $\theta_{2}$ such that $q_{2} t_{n-1} \in I_{\theta_{2}+1}$. Let $s_{0}^{*}=0$ and $0<s_{1}^{*}, \cdots, s_{n-\theta_{2}-1}^{*}<1$ satisfying $q_{2}\left(t_{n-1}+s_{k_{2}}^{*} h_{n}\right)=t_{\theta_{2}+k_{2}}$. Then we have

$$
s_{k_{2}}^{*}:=\left(\frac{t_{\theta_{2}+k_{2}}}{q_{2}}-t_{n-1}\right) / h_{n} \in(0,1), \quad k_{2}=1, \cdots, n-1-\theta_{2},
$$


and define

$$
\begin{aligned}
& B_{n, k_{2}}^{I I}:=\left(\int_{s_{k_{2}-1}^{*}}^{s_{k_{2}}^{*}} b_{2}\left(t_{n-1}+s h_{n}\right) L_{j}\left(\frac{q_{2}\left(t_{n-1}+s h_{n}\right)-t_{\theta_{2}+k_{2}-1}}{h_{\theta_{2}+k_{2}}}\right) L_{i}(s) d s\right)_{1 \leq i, j \leq m+1}{ }^{\prime} \\
& B_{n, 4}^{I I}:=\left(\int_{s_{n-1-\theta_{2}}^{*}}^{1} b_{2}\left(t_{n-1}+s h_{n}\right) L_{j}\left(\frac{q_{2}\left(t_{n-1}+s h_{n}\right)-t_{n-1}}{h_{n}}\right) L_{i}(s) d s\right)_{1 \leq i, j \leq m+1} .
\end{aligned}
$$

In this phase, $U_{n}$ is given by the solution of the linear algebraic system

$$
\begin{aligned}
& \left(M-h_{n} A_{n}-h_{n} B_{n, 2}^{I I}-h_{n} B_{n, 4}^{I I}\right) \mathbf{U}_{n} \\
= & h_{n} \sum_{k_{1}=1}^{n-1-\theta_{1}} B_{n, k_{1}}^{I I} \mathbf{U}_{\theta_{1}+k_{1}}+h_{n} \sum_{k_{2}=1}^{n-1-\theta_{2}} B_{n, k_{2}}^{I I} \mathbf{U}_{\theta_{2}+k_{2}}+G \mathbf{U}_{n-1}+h_{n} \mathbf{f}_{n} .
\end{aligned}
$$

(ii) If $q_{2} t_{n} \leq t_{n-1}$, in this phase, there are two integers $\theta_{2,0}$ and $\theta_{2,1}\left(\theta_{2,0}<\theta_{2,1}\right)$, such that $q_{2} t_{n-1} \in I_{\theta_{2,0}+1}$ and $q_{2} t_{n} \in I_{\theta_{2,1}+1}$. Let $s_{0}^{*}=0,0<s_{1}^{*}, \cdots, s_{\theta_{2,1}-\theta_{2,0}}^{*}<1$ and $s_{\theta_{2,1}-\theta_{2,0}+1}^{*}=1$ satisfying $q_{2}\left(t_{n-1}+s_{k_{2}}^{*} * h_{n}\right)=t_{\theta_{2,0}+k_{2}}$. Then we have

$$
s_{k_{2}}^{*}:=\left(\frac{t_{\theta_{2,0}+k_{2}}}{q_{2}}-t_{n-1}\right) / h_{n} \in(0,1), \quad k_{2}=1, \cdots, \theta_{2,1}-\theta_{2,0}+1,
$$

and define

$$
B_{n, k_{2}}^{I I I}:=\left(\int_{s_{k_{2}-1}^{*}}^{s_{k_{2}}^{*}} b_{2}\left(t_{n-1}+s h_{n}\right) L_{j}\left(\frac{q_{2}\left(t_{n-1}+s h_{n}\right)-t_{\theta_{2,0}+k_{2}-1}}{h_{\theta_{2,0}+k_{2}}}\right) L_{i}(s) d s\right)_{1 \leq i, j \leq m+1} .
$$

In this phase, $\mathbf{U}_{n}$ is the solution of the linear algebraic system

$$
\begin{aligned}
& \left(M-h_{n} A_{n}-h_{n} B_{n, 2}^{I I}\right) \mathbf{U}_{n} \\
= & h_{n} \sum_{k_{2}=1}^{\theta_{2,1}-\theta_{2,0}+1} B_{n, k_{2}}^{I I I} \mathbf{U}_{\theta_{2,0}+k_{2}}+h_{n} \sum_{k_{1}=1}^{n-1-\theta_{1}} B_{n, k_{1}}^{I I} \mathbf{U}_{\theta_{1}+k_{1}}+G \mathbf{U}_{n-1}+h_{n} \mathbf{f}_{n} .
\end{aligned}
$$

(3) If $q_{1} t_{n} \leq t_{n-1},(n \geq 2)$, in this phase, there are two integers $\theta_{1,0}$ and $\theta_{1,1}\left(\theta_{1,0}<\theta_{1,1}\right)$, such that $q_{1} t_{n-1} \in I_{\theta_{1,0}+1}$ and $q_{1} t_{n} \in I_{\theta_{1,1}+1}$. Let $s_{0}^{*}=0,0<s_{1}^{*}, \cdots, s_{\theta_{1,1}-\theta_{1,0}}^{*}<1$ and $s_{\theta_{1,1}-\theta_{1,0}+1}^{*}=1$ satisfying $q_{1}\left(t_{n-1}+s_{k_{1}}^{*} * h_{n}\right)=t_{\theta_{1,0}+k_{1}}$. Then we have

$$
s_{k_{1}}^{*}:=\left(\frac{t_{\theta_{1,0}+k_{1}}}{q_{1}}-t_{n-1}\right) / h_{n} \in(0,1), \quad k_{1}=1, \cdots, \theta_{1,1}-\theta_{1,0}+1,
$$

and define

$$
B_{n, k_{1}}^{I I I}:=\left(\int_{s_{k_{1}-1}^{*}}^{s_{k_{1}}^{*}} b_{1}\left(t_{n-1}+s h_{n}\right) L_{j}\left(\frac{q_{1}\left(t_{n-1}+s h_{n}\right)-t_{\theta_{1,0}+k_{1}-1}}{h_{\theta_{1,0}+k_{1}}}\right) L_{i}(s) d s\right)_{1 \leq i, j \leq m+1} .
$$


Since $q_{1}>q_{2}$, for this reason, $q_{2} t_{n}<t_{n-1}$, the expression of $B_{n, k_{2}}^{I I I}$ is same with (2)(ii). In this phase, $U_{n}$ is given by the solution of the linear algebraic system

$$
\begin{aligned}
& \left(M-h_{n} A_{n}\right) \mathbf{U}_{n} \\
= & h_{n} \sum_{k_{2}=1}^{\theta_{2,1}-\theta_{2,0}+1} B_{n, k_{2}}^{I I I} \mathbf{U}_{\theta_{2,0}+k_{2}}+h_{n} \sum_{k_{1}=1}^{\theta_{1,1}-\theta_{1,0}+1} B_{n, k_{1}}^{I I I} \mathbf{U}_{\theta_{1,0}+k_{1}}+G \mathbf{U}_{n-1}+h_{n} \mathbf{f}_{n} .
\end{aligned}
$$

\section{Convergence analysis of MPDDE with smooth solutions}

In this section, we present and analyze the global convergence and local superconvergence of the DG solution for the multi-pantograph delay differential equation (1.2) with smooth solutions on uniform meshes. Firstly, we briefly discuss the existence and uniqueness of the DG solution which defined by the solutions of the linear algebraic systems (2.7)-(2.10).

For simplicity we select the uniform mesh $J_{h}$ for the interval $J=[0, T]$,

$$
J_{h}=\left\{t_{n}:=n h, n=0,1, \cdots, N\right\}, \quad h=\frac{T}{N} .
$$

\subsection{Existence and uniqueness of the DG solution}

Theorem 3.1. Assume that the given functions $a, b_{1}, b_{2}$ and $f$ in (1.2) are continuous on $J$. Then for any $q_{1}, q_{2} \in(0,1)$ there exists $\bar{h}>0$ (depending on $q_{1}$ and $q_{2}$ ) such that for all $h \in(0, \bar{h})$ each of the linear algebraic systems (2.7)-(2.10) possesses a unique solution $\mathbf{U}_{n} \in \mathbb{R}^{m+1}$.

Proof. Considering the structure of the matrices

$$
M-h_{1} A_{1}-h_{1} B_{1}^{I}-h_{1} B_{2}^{I}, \quad M-h_{n} A_{n}-h_{n} B_{n, 2}^{I I}-h_{n} B_{n, 4}^{I I}, \quad M-h_{n} A_{n}-h_{n} B_{n, 2}^{I I}, \quad M-h_{n} A_{n},
$$

which describe the left-hand sides of the linear algebraic systems (2.7)-(2.10), and the given functions $a, b_{1}$ and $b_{2}$ are in $C(J)$. It is easy to show that $M=\left(M_{i, j}\right),(1 \leq i, j \leq m+1)$ is nonsingular [13]. The non-singularity of $M$ leads to the non-singularity of (2.7)-(2.10) when $h$ is sufficiently small. Therefore, for any $q_{1}, q_{2} \in(0,1)$ there exists a positive $\bar{h}$ so that for all $h \in(0, \bar{h})$ and $1 \leq n \leq N,(2.4)$ defines a unique DG solution $U \in S_{m}^{(-1)}\left(J_{h}\right)$ for Eq. (1.2).

\subsection{Global convergence analysis}

First we need to introduce an appropriate interpolation operator $\Pi_{h}$ which is very important in the convergence analysis. The interpolation operator $\Pi_{h}: C[0,1] \rightarrow S_{m-1}^{(-1)}\left(J_{h}\right)$ is defined by

$$
\begin{aligned}
& \Pi_{h} u\left(t_{n}^{-}\right)=u\left(t_{n}^{-}\right), \\
& \int_{I_{n}} \Pi_{h} u v d t=\int_{I_{n}} u v d t, \quad \forall v \in P_{m-1}\left(I_{n}\right), \quad m \geq 1 .
\end{aligned}
$$


It is obvious that the interpolation operator admits the error estimates

$$
\left\|u-\Pi_{h} u\right\|_{I_{n}, \infty} \leq C h^{m+1}\|u\|_{I_{n}, m+1, \infty} .
$$

To analyze the convergence of finite element approximations, we split the error by writing $e:=u-U=\left(u-\Pi_{h} u\right)+\left(\Pi_{h} u-U\right)=: \xi+\eta$. We can see from (3.2) that the estimates of $\xi$ are available, so we only need to establish the estimate of $\eta$. It then follows readily from the orthogonality property of the DG solution that $\eta$ satisfies

$$
\begin{aligned}
& \int_{I_{n}} \eta^{\prime} v d t+\eta_{n-1}^{+} v_{n-1}^{+} \\
= & \eta_{n-1}^{-} v_{n-1}^{+}+\int_{I_{n}}\left(a(t) e(t)+b_{1}(t) e\left(q_{1} t\right)+b_{2}(t) e\left(q_{2} t\right)\right) v(t) d t, \quad \forall v \in P_{m}\left(I_{n}\right) .
\end{aligned}
$$

Using the integration by parts, we have

$$
\begin{aligned}
& -\int_{I_{n}} \eta v^{\prime} d t+\eta_{n}^{-} v_{n}^{-} \\
= & \eta_{n-1}^{-} v_{n-1}^{+}+\int_{I_{n}}\left(a(t) e(t)+b_{1}(t) e\left(q_{1} t\right)+b_{2}(t) e\left(q_{2} t\right)\right) v(t) d t, \quad \forall v \in P_{m}\left(I_{n}\right),
\end{aligned}
$$

where $\eta_{0}^{-}=0$.

Then we carry out the convergence analysis results of MPDDE (1.2) by using the DG method.

\section{Theorem 3.2. Assume:}

(i) The functions $a, b_{1}, b_{2}$, f describing the MPDDE (1.2) are in $C^{m}(I)$.

(ii) $u \in W^{m+1, \infty}([0, T])$ is the exact solution of the MPDDE (1.2).

(iii) $U \in S_{m}^{(-1)}\left(J_{h}\right)$ is the DG solution defined by (2.4).

(iv) $J_{h}$ is a uniform mesh for $J:=[0, T]$.

We obtain the following optimal global convergence estimates :

$$
\|u-U\|_{\infty} \leq C h^{m+1}\|u\|_{m+1, \infty} .
$$

Proof. In order to prove the estimates (3.5), we first obtain the result

$$
\|u-U\|_{\infty} \leq C\left\|u-\Pi_{h} u\right\|_{\infty}
$$

by using induction, then combine the interpolation error estimate (3.2) we will deduce that (3.5) is true. Let $v=\eta$ in (3.3) and (3.4), summation of these two formulas and using 
the Hölder inequality then yields

$$
\begin{aligned}
& \quad\left|\eta_{n-1}^{+}\right|^{2}+\left|\eta_{n}^{-}\right|^{2} \\
& =2 \int_{I_{n}}\left(a(t) e(t)+b_{1}(t) e\left(q_{1} t\right)+b_{2}(t) e\left(q_{2} t\right)\right) \eta(t) d t+2 \eta_{n-1}^{-} \eta_{n-1}^{+} \\
& \leq 2 \bar{a} \int_{I_{n}}|\xi(t)+\eta(t)||\eta(t)| d t+2 \overline{b_{1}} \int_{I_{n}}\left|\xi\left(q_{1} t\right)+\eta\left(q_{1} t\right)\right||\eta(t)| d t \\
& \quad+2 \overline{b_{2}} \int_{I_{n}}\left|\xi\left(q_{2} t\right)+\eta\left(q_{2} t\right)\right||\eta(t)| d t+\left|\eta_{n-1}^{-}\right|^{2}+\left|\eta_{n-1}^{+}\right|^{2} \\
& \leq \bar{a} \int_{I_{n}}\left(|\xi(t)|^{2}+3|\eta(t)|^{2}\right) d t+\overline{b_{1}} \int_{I_{n}}\left(\left|\xi\left(q_{1} t\right)\right|^{2}+\left|\eta\left(q_{1} t\right)\right|^{2}+2|\eta(t)|^{2}\right) d t \\
& \quad+\overline{b_{2}} \int_{I_{n}}\left(\left|\xi\left(q_{2} t\right)\right|^{2}+\left|\eta\left(q_{2} t\right)\right|^{2}+2|\eta(t)|^{2}\right) d t+\left|\eta_{n-1}^{-}\right|^{2}+\left|\eta_{n-1}^{+}\right|^{2} \\
& \leq\left(3 \bar{a}+2 \overline{b_{1}}+2 \overline{b_{2}}\right) \int_{I_{n}}|\eta(t)|^{2} d t+\bar{a} \int_{I_{n}}|\xi(t)|^{2} d t+\overline{b_{1}} \int_{I_{n}}\left|\eta\left(q_{1} t\right)\right|^{2} d t \\
& \quad+\overline{b_{1}} \int_{I_{n}}\left|\xi\left(q_{1} t\right)\right|^{2} d t+\overline{b_{2}} \int_{I_{n}}\left|\eta\left(q_{2} t\right)\right|^{2} d t+\overline{b_{2}} \int_{I_{n}}\left|\xi\left(q_{2} t\right)\right|^{2} d t+\left|\eta_{n-1}^{-}\right|^{2}+\left|\eta_{n-1}^{+}\right|^{2},
\end{aligned}
$$

where

$$
\bar{a}:=\max _{t \in[0, T]}|a(t)|, \quad \overline{b_{1}}:=\max _{t \in[0, T]}\left|b_{1}(t)\right| \quad \text { and } \quad \overline{b_{2}}:=\max _{t \in[0, T]}\left|b_{2}(t)\right| .
$$

Eliminating the term $\left|\eta_{n-1}^{+}\right|^{2}$, we get

$$
\begin{aligned}
\left|\eta_{n}^{-}\right|^{2} \leq & \left(3 \bar{a}+2 \overline{b_{1}}+2 \overline{b_{2}}\right) \int_{I_{n}}|\eta(t)|^{2} d t+\bar{a} \int_{I_{n}}|\xi(t)|^{2} d t \\
& +\overline{b_{1}} \int_{I_{n}}\left|\xi\left(q_{1} t\right)\right|^{2} d t+\overline{b_{1}} \int_{I_{n}}\left|\eta\left(q_{1} t\right)\right|^{2} d t \\
& +\overline{b_{2}} \int_{I_{n}}\left|\xi\left(q_{2} t\right)\right|^{2} d t+\overline{b_{2}} \int_{I_{n}}\left|\eta\left(q_{2} t\right)\right|^{2} d t+\left|\eta_{n-1}^{-}\right|^{2} .
\end{aligned}
$$

Let $v=\eta^{\prime}(t)\left(t-t_{n-1}\right)$ in (3.3) and we obtain

$$
\begin{aligned}
& \int_{I_{n}}\left|\eta^{\prime}(t)\right|^{2}\left(t-t_{n-1}\right) d t \\
& =\int_{I_{n}} a(t) e(t) \eta^{\prime}(t)\left(t-t_{n-1}\right) d t+\int_{I_{n}} b_{1}(t) e\left(q_{1} t\right) \eta^{\prime}(t)\left(t-t_{n-1}\right) d t \\
& \quad+\int_{I_{n}} b_{2}(t) e\left(q_{2} t\right) \eta^{\prime}(t)\left(t-t_{n-1}\right) d t \\
& \leq \bar{a}\left(\int_{I_{n}}|e(t)|^{2}\left(t-t_{n-1}\right) d t\right)^{\frac{1}{2}} \cdot\left(\int_{I_{n}}\left|\eta^{\prime}(t)\right|^{2}\left(t-t_{n-1}\right) d t\right)^{\frac{1}{2}} \\
& \quad+\overline{b_{1}}\left(\int_{I_{n}}\left|e\left(q_{1} t\right)\right|^{2}\left(t-t_{n-1}\right) d t\right)^{\frac{1}{2}} \cdot\left(\int_{I_{n}}\left|\eta^{\prime}(t)\right|^{2}\left(t-t_{n-1}\right) d t\right)^{\frac{1}{2}}
\end{aligned}
$$




$$
+\overline{b_{2}}\left(\int_{I_{n}}\left|e\left(q_{2} t\right)\right|^{2}\left(t-t_{n-1}\right) d t\right)^{\frac{1}{2}} \cdot\left(\int_{I_{n}}\left|\eta^{\prime}(t)\right|^{2}\left(t-t_{n-1}\right) d t\right)^{\frac{1}{2}} .
$$

Thus,

$$
\begin{aligned}
& \int_{I_{n}}\left|\eta^{\prime}(t)\right|^{2}\left(t-t_{n-1}\right) d t \\
& \leq 2 \bar{a}^{2} \int_{I_{n}}|e(t)|^{2}\left(t-t_{n-1}\right) d t+2{\overline{b_{1}}}^{2} \int_{I_{n}}\left|e\left(q_{1} t\right)\right|^{2}\left(t-t_{n-1}\right) d t \\
& \quad+2{\overline{b_{2}}}^{2} \int_{I_{n}}\left|e\left(q_{2} t\right)\right|^{2}\left(t-t_{n-1}\right) d t \\
& \leq 4 \bar{a}^{2} h \int_{I_{n}}\left(|\xi(t)|^{2}+|\eta(t)|^{2}\right) d t+4{\overline{b_{1}}}^{2} h \int_{I_{n}}\left(\left|\xi\left(q_{1} t\right)\right|^{2}+\left|\eta\left(q_{1} t\right)\right|^{2}\right) d t \\
& \quad+4{\overline{b_{2}}}^{2} h \int_{I_{n}}\left(\left|\xi\left(q_{2} t\right)\right|^{2}+\left|\eta\left(q_{2} t\right)\right|^{2}\right) d t .
\end{aligned}
$$

Let $v=t_{n-1}-t$ in (3.4), we find

$$
\int_{I_{n}} \eta(t) d t-h \eta_{n}^{-}=\int_{I_{n}}\left(a(t) e(t)+b_{1}(t) e\left(q_{1} t\right)+b_{2}(t) e\left(q_{2} t\right)\right)\left(t_{n-1}-t\right) d t .
$$

Then we square both sides of the above equation and obtain

$$
\begin{aligned}
& \quad\left(\int_{I_{n}} \eta(t) d t\right)^{2} \\
& \leq 3 h^{2}\left|\eta_{n}^{-}\right|^{2}+3\left(\int_{I_{n}} a(t) e(t)\left(t_{n-1}-t\right) d t\right)^{2} \\
& \quad+3\left(\int_{I_{n}} b_{1}(t) e\left(q_{1} t\right)\left(t_{n-1}-t\right) d t\right)^{2}+3\left(\int_{I_{n}} b_{2}(t) e\left(q_{2} t\right)\left(t_{n-1}-t\right) d t\right)^{2} \\
& \leq 3 h^{2}\left|\eta_{n}^{-}\right|^{2}+\bar{a}^{2} h^{3} \int_{I_{n}}|e(t)|^{2} d t+\bar{b}_{1}^{2} h^{3} \int_{I_{n}}\left|e\left(q_{1} t\right)\right|^{2} d t+{\overline{b_{2}}}^{2} h^{3} \int_{I_{n}}\left|e\left(q_{2} t\right)\right|^{2} d t \\
& \leq 3 h^{2}\left|\eta_{n}^{-}\right|^{2}+2 \bar{a}^{2} h^{3} \int_{I_{n}}\left(|\xi(t)|^{2}+|\eta(t)|^{2}\right) d t \\
& \quad+2{\overline{b_{1}}}^{2} h^{3} \int_{I_{n}}\left(\left|\xi\left(q_{1} t\right)\right|^{2}+\left|\eta\left(q_{1} t\right)\right|^{2}\right) d t+2{\overline{b_{2}}}^{2} h^{3} \int_{I_{n}}\left(\left|\xi\left(q_{2} t\right)\right|^{2}+\left|\eta\left(q_{2} t\right)\right|^{2}\right) d t .
\end{aligned}
$$

By (3.6)-(3.8), we have following inequalities:

$$
\begin{aligned}
& \left|\eta_{1}^{-}\right|^{2} \leq C\left(\bar{a}+\overline{b_{1}}+\overline{b_{2}}\right) \int_{I_{1}}|\eta(t)|^{2} d t+C\left(\bar{a}+\overline{b_{1}}+\overline{b_{2}}\right) \int_{I_{1}}|\xi(t)|^{2} d t+\left|\eta_{0}^{-}\right|^{2}, \\
& \int_{I_{1}}\left|\eta^{\prime}(t)\right|^{2}\left(t-t_{0}\right) d t \leq C\left(\bar{a}^{2}+{\overline{b_{1}}}^{2}+{\overline{b_{2}}}^{2}\right) h \int_{I_{1}}\left(|\xi(t)|^{2}+|\eta(t)|^{2}\right) d t
\end{aligned}
$$


and

$$
\left(\int_{I_{1}} \eta(t) d t\right)^{2} \leq 3 h^{2}\left|\eta_{1}^{-}\right|^{2}+C\left(\bar{a}^{2}+{\overline{b_{1}}}^{2}+{\overline{b_{2}}}^{2}\right) h^{3} \int_{I_{1}}\left(|\xi(t)|^{2}+|\eta(t)|^{2}\right) d t
$$

where the constant $C$ depend on $q_{1}$ and $q_{2}$, but independent of $h$.

Now we analyze the error estimate on $I_{1}$, we first introduce two useful lemmas.

Lemma 3.1 ([13]). Assume that $I=(a, b)$, for all $\omega \in P_{r}((a, b), \mathbb{R}), r \in \mathbb{N}_{0}$, then

$$
\int_{a}^{b}|\omega|^{2} d t \leq \frac{1}{b-a}\left(\int_{a}^{b} \omega(t) d t\right)^{2}+\frac{1}{2} \int_{a}^{b}(b-t)(t-a)\left|\omega^{\prime}(t)\right|^{2} d t
$$

Lemma 3.2 ([13]). Assume that $I=(a, b)$, a function $\omega$ defined in $(a, b)$. Then the estimate

$$
\|\omega\|_{\infty}^{2} \leq C \log (r+1) \int_{a}^{b}\left|\omega^{\prime}(t)\right|^{2}(t-a) d t+C|\omega(b)|^{2},
$$

holds for all $\omega \in P_{r}((a, b), \mathbb{R}), r \in \mathbb{N}_{0}$. C denotes a positive constant which is independent of the partition.

We combine Lemma 3.1 with (3.10) and obtain

$$
\begin{aligned}
\left(\int_{I_{1}} \eta(t) d t\right)^{2} \leq C & h^{2}\left|\eta_{1}^{-}\right|^{2}+C h^{3}\left(\bar{a}^{2}+{\overline{b_{1}}}^{2}+{\overline{b_{2}}}^{2}\right) \int_{I_{1}}|\xi(t)|^{2} d t \\
+ & C h^{2}\left(\bar{a}^{2}+{\overline{b_{1}}}^{2}+{\overline{b_{2}}}^{2}\right)\left(\int_{I_{1}} \eta(t) d t\right)^{2} \\
+ & C h^{4}\left(\bar{a}^{2}+{\overline{b_{1}}}^{2}+{\overline{b_{2}}}^{2}\right) \int_{I_{1}}\left|\eta^{\prime}(t)\right|^{2}\left(t-t_{0}\right) d t .
\end{aligned}
$$

For sufficiently small $h^{2}\left(\bar{a}^{2}+{\overline{b_{1}}}^{2}+{\overline{b_{2}}}^{2}\right)$, the third term on the right-hand side can be absorbed into the left-hand side. Then

$$
\begin{aligned}
\left(\int_{I_{1}} \eta(t) d t\right)^{2} \leq C & h^{2}\left|\eta_{1}^{-}\right|^{2}+C h^{3}\left(\bar{a}^{2}+{\overline{b_{1}}}^{2}+{\overline{b_{2}}}^{2}\right) \int_{I_{1}}|\xi(t)|^{2} d t \\
+ & C h^{4}\left(\bar{a}^{2}+{\overline{b_{1}}}^{2}+{\overline{b_{2}}}^{2}\right) \int_{I_{1}}\left|\eta^{\prime}(t)\right|^{2}\left(t-t_{0}\right) d t .
\end{aligned}
$$

By Lemma 3.1 and (3.6)-(3.8), we can derive

$$
\begin{aligned}
& \int_{I_{1}}\left|\eta^{\prime}(t)\right|^{2}\left(t-t_{0}\right) d t+\left|\eta_{1}^{-}\right|^{2} \\
\leq & C\left(\bar{a}+\overline{b_{1}}+\overline{b_{2}}\right) \int_{I_{1}}|\xi(t)|^{2} d t+C\left(\bar{a}+\overline{b_{1}}+\overline{b_{2}}\right) \int_{I_{1}}|\eta(t)|^{2} d t+\left|\eta_{0}^{-}\right|^{2} \\
\leq & C\left(\bar{a}+\overline{b_{1}}+\overline{b_{2}}\right) \int_{I_{1}}|\xi(t)|^{2} d t+\frac{C\left(\bar{a}+\overline{b_{1}}+\overline{b_{2}}\right)}{h}\left(\int_{I_{1}} \eta(t) d t\right)^{2} \\
& +C\left(\bar{a}+\overline{b_{1}}+\overline{b_{2}}\right) h \int_{I_{1}}\left|\eta^{\prime}(t)\right|^{2}\left(t-t_{0}\right) d t+\left|\eta_{0}^{-}\right|^{2} .
\end{aligned}
$$


Substituting (3.14) into the above formula yields

$$
\begin{aligned}
& \int_{I_{1}}\left|\eta^{\prime}(t)\right|^{2}\left(t-t_{0}\right) d t+\left|\eta_{1}^{-}\right|^{2} \\
& \leq C h\left(\bar{a}+\overline{b_{1}}+\overline{b_{2}}\right) \int_{I_{1}}\left|\eta^{\prime}(t)\right|^{2}\left(t-t_{0}\right) d t+C h\left(\bar{a}+\overline{b_{1}}+\overline{b_{2}}\right)\left|\eta_{1}^{-}\right|^{2} \\
& \quad+C\left(\bar{a}+\overline{b_{1}}+\overline{b_{2}}\right) \int_{I_{1}}|\xi(t)|^{2} d t+\left|\eta_{0}^{-}\right|^{2} .
\end{aligned}
$$

For $n=1$, we can obtain

$$
\|\eta\|_{I_{1}, \infty}^{2} \leq C \int_{I_{1}}\left|\eta^{\prime}(t)\right|^{2}\left(t-t_{0}\right) d t+C\left|\eta_{1}^{-}\right|^{2} \leq C\left(\bar{a}+\overline{b_{1}}+\overline{b_{2}}\right) \int_{I_{1}}|\xi(t)|^{2} d t
$$

This readily leads to

$$
\|\eta\|_{I_{1}, \infty} \leq C\|\xi\|_{\left[0, t_{1}\right], \infty} .
$$

We now turn to establishing the error estimates for $n \geq 2$. In order to use an induction argument, assume that the estimate

$$
\|\eta\|_{I_{k}, \infty} \leq C\|\xi\|_{\left[0, t_{k}\right], \infty}
$$

is valid for $k=1,2, \cdots, n-1$. Consider the case $k=n$.

$$
\begin{aligned}
& \quad\left(\int_{I_{n}} \eta(t) d t\right)^{2} \\
& \leq C h^{2}\left|\eta_{n}^{-}\right|^{2}+C h^{3}\left(\bar{a}^{2}+{\overline{b_{1}}}^{2}+{\overline{b_{2}}}^{2}\right) \int_{I_{n}}|\xi(t)|^{2} d t+C h^{2}\left(\bar{a}^{2}+{\overline{b_{1}}}^{2}+{\overline{b_{2}}}^{2}\right)\left(\int_{I_{n}} \eta(t) d t\right)^{2} \\
& \quad+C h^{4}\left(\bar{a}^{2}+{\overline{b_{1}}}^{2}+{\overline{b_{2}}}^{2}\right) \int_{I_{n}}\left|\eta^{\prime}(t)\right|^{2}\left(t-t_{n-1}\right) d t \\
& \quad+C h^{3} \overline{b_{1}^{2}} \int_{I_{n}}\left(\left|\xi\left(q_{1} t\right)\right|^{2}+\left|\eta\left(q_{1} t\right)\right|^{2}\right) d t+C h^{3} \overline{b_{2}} \int_{I_{n}}\left(\left|\xi\left(q_{2} t\right)\right|^{2}+\left|\eta\left(q_{2} t\right)\right|^{2}\right) d t \\
& \leq C h^{2}\left|\eta_{n}^{-}\right|^{2}+C h^{3}\left(\bar{a}^{2}+{\overline{b_{1}}}^{2}+{\overline{b_{2}}}^{2}\right) \int_{I_{n}}|\xi(t)|^{2} d t+C h^{2}\left(\bar{a}^{2}+{\overline{b_{1}}}^{2}+{\overline{b_{2}}}^{2}\right)\left(\int_{I_{n}} \eta(t) d t\right)^{2} \\
& \quad+C h^{4}\left(\bar{a}^{2}+{\overline{b_{1}}}^{2}+{\overline{b_{2}}}^{2}\right) \int_{I_{n}}\left|\eta^{\prime}(t)\right|^{2}\left(t-t_{n-1}\right) d t \\
& \quad+C h^{3}{\overline{b_{1}}}^{2} \int_{I_{n}}\left|\xi\left(q_{1} t\right)\right|^{2} d t+C h^{3}{\overline{b_{2}}}^{2} \int_{I_{n}}\left|\xi\left(q_{2} t\right)\right|^{2} d t .
\end{aligned}
$$

For sufficiently small $h^{2}\left(\bar{a}^{2}+{\overline{b_{1}}}^{2}+{\overline{b_{2}}}^{2}\right)$, the third term on the right-hand side also can be absorbed into the left-hand side. We obtain

$$
\begin{aligned}
\left(\int_{I_{n}} \eta(t) d t\right)^{2} \leq C h^{2}\left|\eta_{n}^{-}\right|^{2}+C h^{3}\left(\bar{a}^{2}+{\overline{b_{1}}}^{2}+{\overline{b_{2}}}^{2}\right) \int_{I_{n}}|\xi(t)|^{2} d t+C h^{3} \int_{I_{n}}\left|\xi\left(q_{1} t\right)\right|^{2} d t \\
+C h^{3} \int_{I_{n}}\left|\xi\left(q_{2} t\right)\right|^{2} d t+C h^{4}\left(\bar{a}^{2}+\bar{b}_{1}^{2}+{\overline{b_{2}}}^{2}\right) \int_{I_{n}}\left|\eta^{\prime}(t)\right|^{2}\left(t-t_{n-1}\right) d t,
\end{aligned}
$$


and

$$
\begin{aligned}
& \int_{I_{n}}\left|\eta^{\prime}(t)\right|^{2}\left(t-t_{n-1}\right) d t+\left|\eta_{n}^{-}\right|^{2} \\
& \leq C\left(\bar{a}+\overline{b_{1}}+\overline{b_{2}}\right) \int_{I_{n}}|\xi(t)|^{2} d t+C\left(\bar{a}+\overline{b_{1}}+\overline{b_{2}}\right) \int_{I_{n}}|\eta(t)|^{2} d t+\left|\eta_{n-1}^{-}\right|^{2} \\
& \quad+C \overline{b_{1}} \int_{I_{n}}\left(\left|\xi\left(q_{1} t\right)\right|^{2}+\left|\eta\left(q_{1} t\right)\right|^{2}\right) d t+C \overline{b_{2}} \int_{I_{n}}\left(\left|\xi\left(q_{2} t\right)\right|^{2}+\left|\eta\left(q_{2} t\right)\right|^{2}\right) d t \\
& \leq C\left(\bar{a}+\overline{b_{1}}+\overline{b_{2}}\right) \int_{I_{n}}|\xi(t)|^{2} d t+\frac{C\left(\bar{a}+\overline{b_{1}}+\overline{b_{2}}\right)}{h}\left(\int_{I_{n}} \eta(t) d t\right)^{2} \\
& \quad+C\left(\bar{a}+\overline{b_{1}}+\overline{b_{2}}\right) h \int_{I_{n}}\left|\eta^{\prime}(t)\right|^{2}\left(t-t_{n-1}\right) d t+\left|\eta_{n-1}^{-}\right|^{2} \\
& \quad+C \overline{b_{1}} \int_{I_{n}}\left|\xi\left(q_{1} t\right)\right|^{2} d t+C \overline{b_{2}} \int_{I_{n}}\left|\xi\left(q_{2} t\right)\right|^{2} d t,
\end{aligned}
$$

then

$$
\begin{aligned}
& \int_{I_{n}}\left|\eta^{\prime}(t)\right|^{2}\left(t-t_{n-1}\right) d t+\left|\eta_{n}^{-}\right|^{2} \\
\leq & C h\left(\bar{a}+\overline{b_{1}}+\overline{b_{2}}\right) \int_{I_{n}}\left|\eta^{\prime}(t)\right|^{2}\left(t-t_{n-1}\right) d t+C h\left(\bar{a}+\overline{b_{1}}+\overline{b_{2}}\right)\left|\eta_{n}^{-}\right|^{2} \\
& \quad+C\left(\bar{a}+\overline{b_{1}}+\overline{b_{2}}\right) \int_{I_{n}}|\xi(t)|^{2} d t+\left|\eta_{n-1}^{-}\right|^{2}+C \overline{b_{1}} \int_{I_{n}}\left|\xi\left(q_{1} t\right)\right|^{2} d t+C \overline{b_{2}} \int_{I_{n}}\left|\xi\left(q_{2} t\right)\right|^{2} d t .
\end{aligned}
$$

Iterating the estimate (3.21) yields

$$
\begin{aligned}
& \int_{I_{n}}\left|\eta^{\prime}(t)\right|^{2}\left(t-t_{n-1}\right) d t+\left|\eta_{n}^{-}\right|^{2} \\
& \leq C\left(\bar{a}+\overline{b_{1}}+\overline{b_{2}}\right) \sum_{i=1}^{n} h\left(\int_{I_{i}}\left|\eta^{\prime}(t)\right|^{2}\left(t-t_{i-1}\right) d t+\left|\eta_{i}^{-}\right|^{2}\right) \\
& \quad+C\left(\bar{a}+\overline{b_{1}}+\overline{b_{2}}\right) \sum_{i=1}^{n} \int_{I_{i}}|\xi(t)|^{2} d t+C \sum_{i=1}^{n} \int_{I_{i}}\left|\xi\left(q_{1} t\right)\right|^{2} d t+C \sum_{i=1}^{n} \int_{I_{i}}\left|\xi\left(q_{2} t\right)\right|^{2} d t .
\end{aligned}
$$

For sufficiently small $h\left(\bar{a}+\overline{b_{1}}+\overline{b_{2}}\right)$, Gronwall's Lemma can be applied, this leads to

$$
\begin{aligned}
& \int_{I_{n}}\left|\eta^{\prime}(t)\right|^{2}\left(t-t_{n-1}\right) d t+\left|\eta_{n}^{-}\right|^{2} \\
\leq & \left.C\left(\bar{a}+\overline{b_{1}}+\overline{b_{2}}\right) T \sum_{i=1}^{n}\left(\int_{I_{i}}\left(|\xi(t)|^{2}+\left|\xi\left(q_{1} t\right)\right|^{2}\right) d t+\left|\xi\left(q_{2} t\right)\right|^{2}\right) d t\right) \exp \left(C\left(\bar{a}+\overline{b_{1}}+\overline{b_{2}}\right) T\right) \\
\leq & C\left(\bar{a}+\overline{b_{1}}+\overline{b_{2}}\right) \exp \left(C\left(\bar{a}+\overline{b_{1}}+\overline{b_{2}}\right) T\right) T\left(\|\xi\|_{\infty}^{2}\right) .
\end{aligned}
$$

Using (3.17), (3.18), (3.23), for $1 \leq k \leq N$ we obtain the result

$$
\|u-U\|_{\infty} \leq C\left\|u-\Pi_{h} u\right\|_{\infty} .
$$

This completes the proof. 


\subsection{Local superconvergence analysis}

The following theorem shows that the DG solution of the MPDDE (1.2) has the property of the higher local superconvergence order at the mesh points and Radau II points.

We first recall the definition of the Radau II points in each subinterval $I_{n}$. Let $\left\{p_{n, i}(t)\right.$ : $i \geq 0\}$ denote the set of Legendre polynomials defined on a given subinterval $I_{n}$, and set

$$
u(t)=\sum_{i=0}^{\infty} c_{n, i} p_{n, i}(t), \quad \text { with } \quad c_{n, i}:=(2 i+1) \int_{I_{n}} u(s) \cdot p_{n, i}(s) d s, \quad t \in I_{n} .
$$

Then the zeros of the polynomial $p_{n, m+1}(t)-p_{n, m}(t)$ define the $m+1$ Radau II points in $I_{n}$.

Theorem 3.3. Under the assumptions stated in Theorem 3.2,

(i) Assume that $u \in W^{m+1, \infty}(J)$, the attainable order of the $D G$ solution $U \in S_{m}^{(-1)}\left(J_{h}\right)$ for (1.2) at the mesh points $J_{h} \backslash\{0\}$ of a uniform mesh is given by

$$
\max _{1 \leq n \leq N}\left|\left(u-U_{n}^{-}\right)\left(t_{n}\right)\right| \leq C h^{m+2}\|u\|_{m+1, \infty}, \quad \text { if } m \geq 1 .
$$

If $u \in W^{d, \infty}(J)$ for some $d \geq m+2$, then the term $h^{m+2}$ cannot be replaced by $h^{p}$ with $p>m+2$.

(ii) Assume that $u \in W^{m+2, \infty}(J)$, the attainable order of the $D G$ solution $U \in S_{m}^{(-1)}\left(J_{h}\right)$ for (1.2) at the Radau II points is given by

$$
\left|u\left(t_{n r}\right)-U\left(t_{n r}\right)\right| \leq C h^{m+2}\|u\|_{m+2, \infty}
$$

where $t_{n r}$ stands for any of the Radau II points in $I_{n},(1 \leq n \leq N)$.

Proof. In order to prove the superconvergence of the DG solution $u_{h}$ at the mesh points, we introduce the following auxiliary problem associated with the MPDDE (1.2):

$$
\begin{aligned}
& \phi^{\prime}(t)+a(t) \phi(t)+\tilde{b_{1}}(t) \phi\left(\frac{t}{q_{1}}\right)+\tilde{b_{2}}(t) \phi\left(\frac{t}{q_{2}}\right)=0, \quad t \in\left[0, t_{n}\right), \\
& \phi\left(t_{n}\right)=\alpha:=e_{n}^{-} .
\end{aligned}
$$

Here, $1 \leq n \leq N$ and $\tilde{b_{1}}(t)$ and $\tilde{b_{2}}(t)$ are defined by

$$
\begin{aligned}
& \tilde{b_{1}}(t):= \begin{cases}\frac{1}{q_{1}} b\left(t / q_{1}\right), & 0 \leq t \leq q_{1} t_{n} \\
0, & q_{1} t_{n}<t \leq t_{n}\end{cases} \\
& \tilde{b_{2}}(t):= \begin{cases}\frac{1}{q_{2}} b\left(t / q_{2}\right), & 0 \leq t \leq q_{2} t_{n} \\
0, & q_{2} t_{n}<t \leq t_{n} .\end{cases}
\end{aligned}
$$


From the discontinuity of the function $\tilde{b_{1}}(t)$ and $\tilde{b_{2}}(t)$, we see that the function $\phi^{\prime}(t)$ is discontinuous at the points $q_{1} t_{n}$ and $q_{2} t_{n}$, and we have $\left|\phi^{\prime}\left(q_{1} t_{n}\right)\right| \leq C|\alpha|,\left|\phi^{\prime}\left(q_{2} t_{n}\right)\right| \leq C|\alpha|$. Furthermore, $\left|D^{i} \phi(t)\right| \leq C|\alpha|$, for $t \neq q_{1} t_{n}$ and $t \neq q_{2} t_{n}$. Thus, we use the initial condition $e_{0}^{-}=0$, and obtain the following relation:

$$
\begin{aligned}
& B(e, \phi):= \sum_{j=1}^{n}\left\{\int_{I_{j}}\left(e^{\prime}(s)-a(s) e(s)-b_{1}(s) e\left(q_{1} s\right)-b_{2}(s) e\left(q_{2} s\right)\right) \phi(s) d s+[e]_{j-1} \phi_{j-1}^{+}\right\} \\
&=\sum_{j=1}^{n}\left\{(e \phi)_{j}^{-}-\left(e^{-} \phi^{-}\right)_{j-1}\right.\left.\quad-\int_{I_{j}} e(s)\left(\phi^{\prime}(s)+a(s) \phi(s)+\tilde{b_{1}}(s) \phi\left(s / q_{1}\right)+\tilde{b_{2}}(s) \phi\left(s / q_{2}\right)\right) d s\right\} \\
&=(e \phi)_{n}^{-}=\left|e_{n}^{-}\right|^{2} .
\end{aligned}
$$

Assume now that $\phi_{h} \in S_{m}^{(-1)}\left(J_{h}\right)$ is the (continuous) $m$-th degree piecewise polynomial interpolant of $\phi$ (that is, $\left.\left(\phi-\phi_{h}\right)_{j}^{+}=0\right)$, and let $m \geq 1$. Hence, recalling the orthogonality relations of the DG solution and the exact solution, we have

$$
\begin{aligned}
&\left|e_{n}^{-}\right|^{2}= B(e, \phi)=B\left(e, \phi-\phi_{h}\right) \\
&=\sum_{j=1}^{n} \int_{I_{j}}\left(e^{\prime}(s)-a(s) e(s)-b_{1}(s) e\left(q_{1} s\right)-b_{2}(s) e\left(q_{2} s\right)\right)\left(\phi(s)-\phi_{h}(s)\right) d s \\
&=\sum_{j=1}^{n_{2}^{*}-1} \int_{I_{j}}\left(e^{\prime}(s)-a(s) e(s)-b_{1}(s) e\left(q_{1} s\right)-b_{2}(s) e\left(q_{2} s\right)\right)\left(\phi(s)-\phi_{h}(s)\right) d s \\
& \quad+\int_{I_{n_{2}^{*}}}\left(e^{\prime}(s)-a(s) e(s)-b_{1}(s) e\left(q_{1} s\right)-b_{2}(s) e\left(q_{2} s\right)\right)\left(\phi(s)-\phi_{h}(s)\right) d s \\
& \quad+\sum_{j=n_{2}^{*}+1}^{n_{1}^{*}-1} \int_{I_{j}}\left(e^{\prime}(s)-a(s) e(s)-b_{1}(s) e\left(q_{1} s\right)-b_{2}(s) e\left(q_{2} s\right)\right)\left(\phi(s)-\phi_{h}(s)\right) d s \\
& \quad+\int_{I_{n_{1}^{*}}}\left(e^{\prime}(s)-a(s) e(s)-b_{1}(s) e\left(q_{1} s\right)-b_{2}(s) e\left(q_{2} s\right)\right)\left(\phi(s)-\phi_{h}(s)\right) d s \\
& \quad+\sum_{j=n_{1}^{*}+1}^{n} \int_{I_{j}}\left(e^{\prime}(s)-a(s) e(s)-b_{1}(s) e\left(q_{1} s\right)-b_{2}(s) e\left(q_{2} s\right)\right)\left(\phi(s)-\phi_{h}(s)\right) d s \\
& \leq C\|e\|_{1, \infty,\left[0, t_{n_{2}^{*}-1}\right]}\left\|\phi-\phi_{h}\right\|_{0,1,\left[0, t_{n_{2}^{*}-1}\right]} \\
&+C\left\|_{1, \infty,\left(t_{n_{2}^{*}-1}, t_{n_{2}^{*}}\right)}\right\| \phi-\phi_{h}\left\|_{0,1,\left(t_{n_{2}^{*}-1}, t_{n_{2}^{*}}\right)}+C\right\| e\left\|_{1, \infty,\left[t_{n_{2}^{*}}, t_{n_{1}^{*}-1}\right.}\right\| \phi-\phi_{h} \|_{0,1,\left[t_{n_{2}^{*}}, t_{n_{1}^{*}-1}\right]} \\
&+C\left\|_{1, \infty,\left(t_{n_{1}^{*}-1}, t_{n_{1}^{*}}\right)}\right\| \phi-\phi_{h}\left\|_{0,1,\left(t_{n_{1}^{*}-1}, t_{n_{1}^{*}}\right)}+C\right\| e\left\|_{1, \infty,\left[t_{n_{1}^{*}}, t_{n}\right]}\right\| \phi-\phi_{h} \|_{0,1,\left[t_{n_{1}^{*}}, t_{n}\right]} \quad(3.29) \\
& \leq C h^{m} h^{m+1}\|u\|_{m+1, \infty,\left[0, t_{n_{2}^{*}-1}\right]}\left|e_{n}^{-}\right| \\
&\left.+C h^{m} h\|u\|_{m+1, \infty,\left(t_{n_{2}^{*}-1}, t_{n_{2}^{*}}\right)}\right)\left.\phi^{\prime}(t)\right|_{\infty,\left(t_{n_{2}^{*}-1}, t_{n_{2}^{*}}\right)} \operatorname{meas}\left(\left(t_{n_{2}^{*}-1}, t_{n_{2}^{*}}\right)\right)
\end{aligned}
$$




$$
\begin{aligned}
&\left.+C h^{m} h^{m+1}\|u\|_{m+1, \infty,\left[t_{n_{2}^{*},}, t_{1}^{*}-1\right.}\right] e_{n}^{-} \mid \\
&+C h^{m} h\|u\|_{m+1, \infty,\left(t_{n_{1}^{*}-1}, t_{n_{1}^{*}}^{*}\right.}\left|\phi^{\prime}(t)\right|_{\infty,\left(t_{n_{1}^{*}-1}, t_{n_{1}^{*}}\right)} \operatorname{meas}\left(\left(t_{n_{1}^{*}-1}, t_{n_{1}^{*}}\right)\right) \\
&+C h^{m} h^{m+1}\|u\|_{m+1, \infty,\left[t_{n_{1}^{*}}, t_{n}\right]}\left|e_{n}^{-}\right| \\
&\left.\leq C h^{m+2}\|u\|_{m+1, \infty,\left[0, t_{n}\right]}\right] e_{n}^{-} \mid .
\end{aligned}
$$

Here $q_{1} t_{n} \in\left(t_{n_{1}^{*}-1}, t_{n_{1}^{*}}\right)$ and $q_{2} t_{n} \in\left(t_{n_{2}^{*}-1}, t_{n_{2}^{*}}\right)$. Since $q_{1} t_{n}>q_{2} t_{n}$, we have $t_{n_{1}^{*}} \geq t_{n_{2}^{*}}$ and $n_{1}^{*} \geq n_{2}^{*}$. We consider the case of $n_{2}^{*}<n_{1}^{*}-1$ above, all the third terms of (3.31)-(3.29) are omitted when $n_{2}^{*}=n_{1}^{*}-1$ and $n_{2}^{*}=n_{1}^{*}$. The estimate (3.31) implies that

$$
\left|e_{n}^{-}\right| \leq C h^{m+2}\|u\|_{m+1, \infty}, \quad n=1, \cdots, N .
$$

This means that we have established the desired result (3.25) for all $m \geq 1$. The proof of (3.26) is similar to one delay term (see [14, pp. 2670]). We leave it to the reader.

\section{Convergence analysis of weakly singular MPDDE}

The convergence results in Theorems 3.2 and 3.3 are valid for solutions that sufficiently smooth in $[0, T]$. However, this regularity assumption is unrealistic if $f$ (the right hand side term) is weakly singular. Since solutions of MPDDEs (1.2) have strong start-up singularities [20] due to the presence of $f$. In this section we show that despite the solution has a singularity at $t=0$, we can also obtain the algebraic convergence with graded meshes by the DG method.

We suppose the singular term $f$ has the form

$$
f(t)=f_{1}(t)+t^{\beta} f_{2}(t), \quad \beta \in(0,1) .
$$

If the partition $\left\{I_{n}\right\}_{n=1}^{N}$ are given by

$$
\hat{J}_{n}=\left\{t_{n}:=\left(\frac{n}{N}\right)^{r} T, n=0, \cdots, N\right\},
$$

where the grading exponent $r \in R$ will always be assumed to satisfy $r>1$, then $\hat{J_{n}}$ is called the graded mesh. For any such mesh we have $0<h_{1}<\cdots<h_{N}$.

As a special case of [20] in which $m_{n}=m$, the following lemmas describe the approximation properties of the interpolant $\Pi_{h} u$ under graded meshes.

Lemma 4.1. Let $\hat{J}_{n}$ be a graded mesh for the given interval $[0, T]$, set $I_{n}=\left(t_{n-1}, t_{n}\right), h_{n}=t_{n}-t_{n-1}$, $m \in \mathbb{N}_{0}$, and $u \in W^{s_{0}+1, \infty}\left(I_{n}\right)$, for some $s_{0} \geq 0$. Then we have

$$
\left\|u-\Pi_{h} u\right\|_{I_{n}, \infty}^{2} \leq C\left(\frac{h_{n}}{2}\right)^{2 s+2} \frac{\Gamma(m+1-s)}{\Gamma(m+1+s)}\|u\|_{I_{n}, s+1, \infty}^{2}
$$

for any real $s$ with $0 \leq s \leq \min \left(m, s_{0}\right)$, and

$$
\|u-U\|_{\infty} \leq C\left\|u-\Pi_{h} u\right\|_{\infty} .
$$


Lemma 4.2. Let $\gamma=1+\beta$ and $\hat{J}_{n}$ be a graded mesh of $[0, T]$. The constant $C, d>0$ depending only on the analyticity constants of $a, b_{1}, b_{2}, f_{1}$ and $f_{2}$, such that the solution $u$ of MPDDE (1.2) satisfies

$$
\begin{aligned}
& \|u\|_{I_{1}, 1, \infty}^{2} \leq C, \\
& \left|u^{(s)}(t)\right| \leq C d^{s} \Gamma(s+1) t^{\gamma-s}, \quad t \in(0, T], \quad s \in N .
\end{aligned}
$$

The next result establishes the convergence of the DG method for weakly singular MPDDEs.

Theorem 4.1. Assume:

(i) The functions $a, b_{1}, b_{2}, f_{1}, f_{2}$ describing the MPDDE (1.2) are in $C^{m}(I)$.

(ii) $u \in W^{\gamma, \infty}\left(\left[0, t_{1}\right]\right) \cup W^{m+1, \infty}\left(\left(t_{1}, T\right]\right)$ is the exact solution of MPDDE (1.2).

(iii) $U \in S_{m}^{(-1)}\left(J_{h}\right)$ is the $D G$ solution defined by (2.4).

(iv) $\hat{J}_{n}$ is a graded mesh for $J:=[0, T]$, with grading exponent $r \geq m+1$.

We obtain the following optimal global convergence estimates :

$$
\|u-U\|_{\infty} \leq C h^{m+1}, \quad(h:=T / N) .
$$

Proof. Since the solution has singularity at $t_{1}=0$, we particularly bound the errors at the first interval $\hat{J}_{1}$. From Lemma 4.1,

$$
\|u-U\|_{\infty}^{2} \leq C \max _{1 \leq n \leq N+1} e_{n}^{2}
$$

with

$$
e_{n}^{2}=C\left(\frac{h_{n}}{2}\right)^{2 s+2} \frac{\Gamma(m+1-s)}{\Gamma(m+1+s)}\|u\|_{I_{n}, s+1, \infty}^{2} .
$$

For the first subinterval $\hat{J}_{1}$, since $s=0$, we have from (4.4a)

$$
\left\|e_{1}\right\|_{\infty} \leq C h_{1} .
$$

Since $h_{1}=\left(\frac{1}{N}\right)^{r} T, r \geq m+1$, then

$$
\left\|e_{1}\right\|_{\infty} \leq C h^{m+1} .
$$

From (4.4b), the regularity exponents $s$ can be chosen arbitrary large for $n=2, \cdots, N$. The solution become smooth after a non-smooth initial phase. For $n \geq 2$ the convergence analysis can be proved by using similar techniques in Theorem 3.2.

The following theorem gives the result of the local superconvergence.

Theorem 4.2. Under the same assumptions in Theorem 4.1. 
(i) The attainable superconvergence order of DG solution at the mesh points of the graded mesh is given by

$$
\max _{1 \leq n \leq N+1}\left|\left(u-U_{n}^{-}\right)\left(t_{n}\right)\right| \leq C h^{m+2}, \quad \text { if } m \geq 1 .
$$

(ii) The attainable superconvergence order of DG solution at the Radau II points of the graded mesh is given by

$$
\left|u\left(t_{n r}\right)-U\left(t_{n r}\right)\right| \leq C h^{m+2}
$$

Proof. The proof here is similar to the proof of Theorem 3.3. We leave it to the reader.

Remark 4.1. The DG method can also be used to solve system of MPDDEs with smooth and weakly singular solutions. By constructing high-dimensional linear algebraic systems and similar to (2.7)-(2.10), we can obtain the global convergence and local superconvergence both on uniform meshes and on graded meshes (see Example 5.3 of Section $5)$.

Remark 4.2. We can extend the DG method for MPDDEs to the general nonlinear case. In our future work, we will consider DG method for nonlinear MPDDEs including the nonlinear delay functions and the nonlinear problems.

\section{Numerical experiments}

In this section, we present several numerical experiments to verify the accuracy and efficiency of the theoretical analysis. We use the following notations:

$$
\begin{array}{ll}
e r g=|| u-U \|_{\infty}, & R=\frac{\log \left(\operatorname{erg}_{N 1} / \operatorname{erg}_{N 2}\right)}{\log \left(h_{N 1} / h_{N 2}\right)}, \\
e r n=\max _{1 \leq n \leq N}\left|u\left(t_{n}\right)-U\left(t_{n}\right)\right|, & R_{n}=\frac{\log \left(e r n_{N 1} / e r n_{N 2}\right)}{\log \left(h_{N 1} / h_{N 2}\right)}, \\
e r r=\max _{\substack{1 \leq n \leq N \\
1 \leq r \leq m+1}}\left|u\left(t_{n r}\right)-U\left(t_{n r}\right)\right|, & R_{r}=\frac{\log \left(e r r_{N 1} / e r r_{N 2}\right)}{\log \left(h_{N 1} / h_{N 2}\right)},
\end{array}
$$

where $t_{n r}$ denote Radau II points, and $t_{n}$ denote the nodal points.

Example 5.1. We consider the following multi-pantograph equation

$$
\begin{aligned}
& u^{\prime}(t)=-u(t)+b_{1}(t) u(0.5 t)+b_{2}(t) u(0.25 t), \quad 0<t \leq 1, \\
& u(0)=1,
\end{aligned}
$$

where $b_{1}(t)=-e^{-0.5 t} \sin (0.5 t), b_{2}(t)=-2 e^{-0.75 t} \cos (0.5 t) \sin (0.25 t)$. 
The exact solution $u(t)=e^{-t} \cos (t)$ is analytic on $[0,1]$. We choose uniform meshes $J_{h}$ with mesh size $h=1 / N,(N=16,32,64, \cdots)$. Numerical results are obtained by the piecewise linear DG approximation $(m=1)$ and by the piecewise quadratic DG approximation $(m=2)$.

(1) Errors of piecewise linear DG solutions $(m=1)$ :

Table 1: Errors of piecewise linear DG solution.

\begin{tabular}{||ccccccc||}
\hline$N$ & erg & $\mathrm{R}$ & ern & $\mathrm{R}_{n}$ & err & $\mathrm{R}_{r}$ \\
\hline 16 & $3.6144 \mathrm{e}-04$ & & $3.1019 \mathrm{e}-06$ & & $2.7784 \mathrm{e}-06$ & \\
32 & $9.0804 \mathrm{e}-04$ & 1.9929 & $3.9670 \mathrm{e}-07$ & 2.9671 & $3.4883 \mathrm{e}-07$ & 2.9936 \\
64 & $2.2763 \mathrm{e}-05$ & 1.9961 & $4.9822 \mathrm{e}-08$ & 2.9932 & $4.3661 \mathrm{e}-08$ & 2.9981 \\
128 & $5.6994 \mathrm{e}-06$ & 1.9978 & $6.2474 \mathrm{e}-09$ & 2.9955 & $5.4600 \mathrm{e}-09$ & 2.9994 \\
\hline
\end{tabular}

(2) Errors of piecewise quadratic DG solutions $(m=2)$ :

Table 2: Errors of piecewise quadratic DG solution.

\begin{tabular}{||ccccccc||}
$N$ & erg & $\mathrm{R}$ & ern & $\mathrm{R}_{n}$ & err & $\mathrm{R}_{r}$ \\
8 & $4.0397 \mathrm{e}-05$ & & $6.6310 \mathrm{e}-07$ & & $2.3693 \mathrm{e}-07$ & \\
16 & $5.4428 \mathrm{e}-06$ & 2.8918 & $4.3674 \mathrm{e}-08$ & 3.9244 & $1.4603 \mathrm{e}-08$ & 3.9834 \\
32 & $7.0587 \mathrm{e}-07$ & 2.9469 & $2.8009 \mathrm{e}-09$ & 3.9628 & $9.2321 \mathrm{e}-10$ & 4.0202 \\
64 & $8.9860 \mathrm{e}-08$ & 2.9737 & $1.7731 \mathrm{e}-10$ & 3.9816 & $5.6566 \mathrm{e}-11$ & 4.0287 \\
\hline
\end{tabular}

We conclude from Tables 1-2 that

$$
\begin{aligned}
& \|u-U\|_{\infty}=\mathcal{O}\left(h^{m+1}\right), \\
& \max _{\substack{1 \leq n \leq N \\
1 \leq r \leq m+1}}\left|u\left(t_{n r}\right)-U\left(t_{n r}\right)\right|=\mathcal{O}\left(h^{m+2}\right), \\
& \max _{1 \leq n \leq N}\left|u\left(t_{n}\right)-U_{n}\right|=\mathcal{O}\left(h^{m+2}\right), \quad m=1,2 .
\end{aligned}
$$

This confirms the correctness of theoretical results.

Example 5.2. We consider the MPDDE with weakly singular solution

$$
\begin{aligned}
& u^{\prime}(t)=-u(t)+\frac{1}{2} u\left(\frac{1}{3} t\right)+\frac{1}{2} u\left(\frac{1}{4} t\right)+f(t), \quad 0<t \leq 1, \\
& u(0)=1 .
\end{aligned}
$$

Where $f(t)$ is set to make the exact solution $u(t)=t^{1.5} e^{-t}$.

It is obvious that the function $f(t)$ has the form (4.1) with $\beta=0.5$, the solution only satisfies $u \in W^{1.5, \infty}$. Numerical results are obtained by the piecewise quadratic DG approximation with graded meshes. 
Table 3: Errors of piecewise quadratic DG solution, $r=3$.

\begin{tabular}{||ccccccc||}
\hline$N$ & erg & $\mathrm{R}$ & ern & $\mathrm{R}_{n}$ & err & $\mathrm{R}_{r}$ \\
\hline 60 & $4.3317 \mathrm{e}-07$ & & $1.6985 \mathrm{e}-09$ & & $3.8711 \mathrm{e}-09$ & \\
120 & $5.7381 \mathrm{e}-08$ & 2.9520 & $1.0123 \mathrm{e}-10$ & 4.1182 & $2.4176 \mathrm{e}-10$ & 4.0500 \\
180 & $1.7279 \mathrm{e}-08$ & 2.9805 & $2.3632 \mathrm{e}-11$ & 3.8128 & $4.8626 \mathrm{e}-11$ & 3.9827 \\
240 & $7.3663 \mathrm{e}-09$ & 2.9780 & $7.8241 \mathrm{e}-12$ & 3.8611 & $1.4979 \mathrm{e}-11$ & 4.1129 \\
\hline
\end{tabular}

Table 4: Errors of piecewise quadratic DG solution, $r=3.5$.

\begin{tabular}{||ccccccc||}
\hline$N$ & $e r g$ & $\mathrm{R}$ & ern & $\mathrm{R}_{n}$ & err & $\mathrm{R}_{r}$ \\
\hline 60 & $6.7591 \mathrm{e}-07$ & & $1.7801 \mathrm{e}-09$ & & $6.0691 \mathrm{e}-09$ & \\
120 & $8.9830 \mathrm{e}-08$ & 2.9116 & $1.7162 \mathrm{e}-10$ & 3.8747 & $3.8740 \mathrm{e}-10$ & 3.9696 \\
180 & $2.7287 \mathrm{e}-08$ & 2.9386 & $4.2109 \mathrm{e}-11$ & 3.8653 & $7.7034 \mathrm{e}-11$ & 3.9836 \\
240 & $1.1632 \mathrm{e}-08$ & 2.9639 & $1.1637 \mathrm{e}-11$ & 4.1705 & $2.4437 \mathrm{e}-11$ & 3.9910 \\
\hline
\end{tabular}

For $m=2$, we conclude from Tables 3-4 that

$$
\begin{aligned}
& \|u-U\|_{\infty}=\mathcal{O}\left(h^{3}\right), \\
& \max _{\substack{1 \leq n \leq N \\
1 \leq r \leq m+1}}\left|u\left(t_{n r}\right)-U\left(t_{n r}\right)\right|=\mathcal{O}\left(h^{4}\right), \\
& \max _{1 \leq n \leq N}\left|u\left(t_{n}\right)-U_{n}\right|=\mathcal{O}\left(h^{4}\right) .
\end{aligned}
$$

Even though the solution $u \in W^{1.5, \infty},(1.5<m)$, we still can obtain the optimal global convergence and local superconvergence with graded meshes.

Example 5.3. Consider the system of MPDDEs:

$$
\begin{aligned}
& u_{1}^{\prime}(t)=-\frac{1}{2} u_{2}(t)-u_{1}(t)+\frac{1}{5} u_{1}\left(\frac{1}{2} t\right)+\frac{1}{6} u_{2}\left(\frac{1}{3} t\right)+f_{1}(t), \\
& u_{2}^{\prime}(t)=-\frac{1}{3} u_{1}(t)-\frac{1}{4} u_{2}(t)+\frac{1}{10} u_{2}\left(\frac{1}{2} t\right)+\frac{3}{10} u_{1}\left(\frac{1}{3} t\right)+f_{2}(t), \quad 0<t \leq 1,
\end{aligned}
$$

subject to the initial conditions $u_{1}(0)=0, u_{2}(0)=1$.

We set $f_{1}(t)$ and $f_{2}(t)$ to make the exact solutions $u_{1}(t)=t^{1.5}, u_{2}(t)=\cos (t)$.

We approximate $u_{1}$ and $u_{2}$ by the piecewise quadratic DG method. Due to the weakly singular solution $u_{1}$, we select the graded meshes. In each subinterval $I_{n},(n=1, \cdots, N)$, let

$$
U_{n}^{1}(t)=\sum_{j=1}^{m+1} u_{n, j}^{1} l_{n, j}(t)=\sum_{j=1}^{m+1} u_{n, j}^{1} L_{j}\left(\frac{t-t_{n-1}}{h_{n}}\right)
$$

and

$$
U_{n}^{2}(t)=\sum_{j=1}^{m+1} u_{n, j}^{2} l_{n, j}(t)=\sum_{j=1}^{m+1} u_{n, j}^{2} L_{j}\left(\frac{t-t_{n-1}}{h_{n}}\right)
$$


Table 5: Errors for quadratic DG approximation of $u_{1}, r=3$.

\begin{tabular}{||ccccccc||}
\hline$N$ & erg & $\mathrm{R}$ & ern & $\mathrm{R}_{n}$ & err & $\mathrm{R}_{r}$ \\
\hline 60 & $5.5457 \mathrm{e}-07$ & & $3.2617 \mathrm{e}-10$ & & $2.0694 \mathrm{e}-09$ & \\
120 & $6.9780 \mathrm{e}-08$ & 3.0270 & $1.9784 \mathrm{e}-11$ & 4.0926 & $1.3081 \mathrm{e}-10$ & 4.0323 \\
180 & $9.0738 \mathrm{e}-08$ & 3.0133 & $5.0610 \mathrm{e}-12$ & 3.8856 & $2.5328 \mathrm{e}-11$ & 4.0773 \\
240 & $8.7538 \mathrm{e}-09$ & 3.0126 & $1.7489 \mathrm{e}-13$ & 3.8714 & $8.0487 \mathrm{e}-12$ & 4.0044 \\
\hline
\end{tabular}

Table 6: Errors for quadratic DG approximation of $u_{2}, r=3$.

\begin{tabular}{||ccccccc||}
\hline$N$ & erg & $\mathrm{R}$ & ern & $\mathrm{R}_{n}$ & err & $\mathrm{R}_{r}$ \\
\hline 60 & $3.2020 \mathrm{e}-04$ & & $1.2715 \mathrm{e}-06$ & & $2.6127 \mathrm{e}-06$ & \\
120 & $1.6670 \mathrm{e}-05$ & 2.9175 & $2.1139 \mathrm{e}-08$ & 4.0443 & $4.3867 \mathrm{e}-08$ & 4.0346 \\
180 & $5.0654 \mathrm{e}-07$ & 2.9746 & $1.6053 \mathrm{e}-10$ & 4.1551 & $3.9785 \mathrm{e}-10$ & 4.0040 \\
240 & $6.4891 \mathrm{e}-08$ & 2.9916 & $1.1629 \mathrm{e}-11$ & 3.8217 & $2.8813 \mathrm{e}-11$ & 3.8220 \\
\hline
\end{tabular}

be DG approximations of $u_{1}$ and $u_{2}$, respectively. We obtain the unknown vector

$$
U_{n}=\left(u_{n, 1}^{1}, \cdots, u_{n, m+1}^{1}, u_{n, 1}^{2}, \cdots, u_{n, m+1}^{2}\right)^{T} \in \mathbb{R}^{2 m+2} .
$$

From Tables 5-6 above, we obtain the optimal global convergence and local superconvergence by using the piecewise quadratic DG method to solve the system of the MPDDEs.

\section{Concluding remarks}

In this paper, the DG methods are employed to solve MPDDEs with smooth and weakly singular solutions. We obtain the global convergence and local superconvergence both on uniform meshes and graded meshes. Numerical experiments show the efficiency of the DG method for solving MPDDEs.

We remark that the current technique can be extended to general nonuniform meshes and the same global convergence and local nodal superconvergence of DG approximations can be obtained for smooth solutions of MPDDEs. But when the source term is singular, special nonuniform meshes relating to the singularities are necessary, which we used graded meshes this paper. Some other special nonuniform meshes relating to the singularities, such as geometric meshes, are also can be used to get the same results. The proof of the global convergence and local superconvergence are similar with those of the graded meshes.

The following two problems remain to be addressed in future research work:

1. Superconvergence analysis of the postprocessing acceleration techniques for DG solutions of MPDDEs;

2. Analysis of the continuous Galerkin method for MPDDEs; 
3. Analysis of the DG method for nonlinear system of MPDDEs.

\section{Acknowledgements}

The authors were supported by the Natural Science Foundation of China (No. 11571027), the International Research Cooperation Seed of Beijing University of Technology (No. 2018B32), Science and Technology Projects of Beijing Education Commission Foundatio (No. KM201510005032), and the 16th graduate science and technology fund of Beijing university of technology (No. ykj-2017-00127).

\section{References}

[1] E. ISHIWATA, On the attainable order of collocation methods for the neutral functional-differential equations with proportional delays, Comput., 64 (2000), pp. 207-222.

[2] D. Li AND M. Z. LIU, Runge-Kutta methods for the multi-pantograph delay equation, Appl. Math. Comput., 163 (2005), pp. 383-395.

[3] M. SEZER, S. YALCINBAS AND N. SAHIN, Approximate solution of multi-pantograph equation with variable coeffcients, J. Comput. Appl. Math., 241 (2008), pp. 406-416.

[4] H. BRUNNER, Collocation methods for pantograph-type Volterra functional equations with multiple delays, Comput. Meth. Appl. Math., 8 (2008), pp. 207-222.

[5] Z. H. YU, Variational iteration method for solving the multi-pantograph delay equation, Phys. Lett. A, 372 (2008), pp. 6475-6479.

[6] X. L. FENG, An analytic study on the multi-pantograph delay equations with variable coefficients, Soc. Sci. Math., 56 (2013), pp. 205-215.

[7] F. Z. GENG AND S. P. QIAN, Solving singularly perturbed multipantograph delay equations based on the reproducing kernel method, Abstr. Appl. Anal., 3 (2014), pp. 1-6.

[8] I. Komashynska, M. Alsmadi, A. AlHabAhBeH And A. Ateiwi, Analytical approximate solutions of systems of multi-pantograph delay differential equations using residual power-series method, Aust. J. Basic Appl. Sci., 8 (2014), pp. 664-675.

[9] S. DAVAEIFAR AND J. RASHIDINIA, Solution of a system of delay differential equations of multipantograph type, J. Taibah. Univ. Sc., 11 (2017), pp. 1141-1157.

[10] W. S. ZHENG, Y. P. CHEN AND Y. Q HUANG, Convergence analysis of Legendre-collocation spectral methods for second order Volterra integro-differential equation with delay, Adv. Appl. Math. Mech., 11 (2019), pp. 486-500.

[11] S. Sedaghat, S. Nemati AND Y. ORdokHani, Convergence analysis of spectral method for neutral multi-pantograph equations, Ira. J. Sci. Tech. A, 2 (2018), pp. 1-8.

[12] P. LASAINT AND P. A. RAVIART, On a finite element method for solving the neutron transport equation, Mathematical Aspects of Finite Elements in Partial Differential Equations, Academic Press, New York, San Francisco, London, (1974), pp. 89-123.

[13] H. BRUnNer, Q. M. HuAng AND H. H. XIE, Discontinuous Galerkin methods for delay differential equations of pantograph type, SIAM J. Numer. Anal., 48 (2010), pp. 1944-1967.

[14] Q. M. HuAnG, H. H. XIE AND H. BRUnNER, Superconvergence of discontinuous Galerkin solutions for delay differential equations of pantograph type, SIAM J. Sci. Comput., 33 (2011), pp. 2664-2684. 
[15] Q. M. HuAng, H. H. XIE AND H. BRUnNeR, The hp discontinuous Galerkin method for delay differential equations with nonlinear vanishing delay, SIAM J. Sci. Comput., 35 (2013), pp. 16041620.

[16] X. X. XU, Q. M. HuANG AND H. T. CHEN, Local superconvergence of continuous Galerkin solutions for delay differential equations of pantograph type, J. Comput. Math., 24 (2016), pp. 186-199.

[17] Q. M. HuANG, X. X. XU AND H. BRUnNER, Continuous Galerkin methods on quasi-geometric meshes for delay differential equations of pantograph type, Discrete. Cont. Dyn. A, 36 (2016), pp. 5423-5443.

[18] Q. M. HuANG, K. JiAng AND X. X. XU, Postprocessing of continuous Galerkin solutions for delay differential equations with nonlinear vanishing delay, Int. J. Numer. Anal. Mod., 16 (2019), pp. 718-730.

[19] Q. LIN AND J. LIN, Finite Element Methods: Accuracy and Improvement, Science Press, Beijing, 2006.

[20] H. BRUNNER AND D. DCHÖTZAU, Hp-discontinuous Galerkin time-stepping for Volterra integro differential equations, SIAM J. Numer. Anal., 44 (2006), pp. 224-245.

[21] D. F. LI AND C. J. ZHANG, Nonlinear stability of discontinuous Galerkin methods for delay differential equations, Appl. Math. Lett., 23 (2010), pp. 457-461. 